\title{
V-singular values of rectangular tensors and their applications
}

\author{
Jun $\mathrm{He}^{1 *}$, Yanmin Liu ${ }^{1}$, Guangjun $\mathrm{Xu}^{1}$ and Gang Liu ${ }^{2}$
}

"Correspondence:

hejunfan1@163.com

'School of Mathematics, Zunyi Normal College, Zunyi, P.R. China

Full list of author information is

available at the end of the article

\begin{abstract}
The positive definiteness of rectangular tensors has wide applications in solid mechanics and quantum physics. By modifying the existing definition of singular value for rectangular tensors, some $\mathrm{V}$-singular value inclusion sets for rectangular tensors with positive diagonal entries are established to provide verifiable sufficient conditions for the positive definiteness of rectangular tensors. In addition, an upper bound for the largest $\mathrm{V}$-singular value of nonnegative rectangular tensors is provided.
\end{abstract}

MSC: 15A18; 15A42; 15A69

Keywords: Rectangular tensors; V-singular value; Inclusion set; Positive definiteness

\section{Introduction}

Let $\mathbb{R}(\mathbb{C}$ ) be the real (complex) field, $p, q, m, n$ be positive integers, $m, n \geq 2$, $[n]=$ $\{1,2, \ldots, n\}$. A $(p, q)$ th order $(m \times n)$-dimensional real rectangular tensor, denoted by $\mathcal{A}=\left(a_{i_{1} \ldots i_{j} j_{1} \ldots j_{q}}\right) \in \mathbb{R}^{[p ; q ; m ; n]}$, is defined as follows:

$$
a_{i_{1} \ldots i_{p} j_{1} \ldots j_{q}} \in \mathbb{R}, \quad i_{1}, \ldots, i_{p} \in[m], j_{1}, \ldots, j_{q} \in[n]
$$

When $p=q=1, \mathcal{A}$ is simply a real $m \times n$ rectangular matrix.

$\mathcal{A}$ is called nonnegative if $a_{i_{1} \ldots i_{p} j_{1} \ldots j_{q}} \geq 0$, denoted by $\mathcal{A} \in \mathbb{R}_{+}^{[p ; q ; m ; n]}$. We say that $\mathcal{A}$ is a real partially symmetric rectangular tensor if $a_{i_{1} \ldots i_{p} j_{1} \ldots j_{q}}$ is invariant under any permutation of indices among $i_{1}, \ldots, i_{p}$ and any permutation of indices among $j_{1}, \ldots, j_{q}$, i.e.,

$$
a_{\pi\left(i_{1} \ldots i_{p}\right) \sigma\left(j_{1} \ldots j_{q}\right)}=a_{i_{1} \ldots i_{p} j_{1} \ldots j_{q}}, \quad \pi \in S_{p}, \sigma \in S_{q}
$$

where $S_{r}$ is the permutation group of $r$ indices.

For any vectors $x \in \mathbb{C}^{m}, y \in \mathbb{C}^{n}$, let $\mathcal{A} x^{p-1} y^{q}$ be a vector in $\mathbb{C}^{m}$ such that

$$
\left(\mathcal{A} x^{p-1} y^{q}\right)_{i}=\sum_{i_{2}, \ldots, i_{p}=1}^{m} \sum_{j_{1}, \ldots, j_{q}=1}^{n} a_{i i_{2} \ldots i_{p} j_{1} \ldots j_{q}} x_{i_{2}} \cdots x_{i_{p}} y_{j_{1}} \cdots y_{j_{q}},
$$

where $i \in[m]$. Let $\mathcal{A} x^{p} y^{q-1}$ be a vector in $\mathbb{C}^{n}$ such that

$$
\left(\mathcal{A} x^{p} y^{q-1}\right)_{j}=\sum_{i_{1}, \ldots, i_{p}=1}^{m} \sum_{j_{2}, \ldots, j_{q}=1}^{n} a_{i_{1} i_{2} \ldots i_{p} j j_{2} \ldots j_{q}} x_{i_{1}} \cdots x_{i_{p}} y_{j_{2}} \cdots y_{j_{q}}
$$

(c) The Author(s) 2019. This article is distributed under the terms of the Creative Commons Attribution 4.0 International License (http://creativecommons.org/licenses/by/4.0/), which permits unrestricted use, distribution, and reproduction in any medium, provided you give appropriate credit to the original author(s) and the source, provide a link to the Creative Commons license, and indicate if changes were made. 
where $j \in[n]$. If there exist a number $\lambda \in \mathbb{C}$, vectors $x \in \mathbb{C}^{m} \backslash\{0\}$, and $y \in \mathbb{C}^{n} \backslash\{0\}$ such that

$$
\left\{\begin{array}{l}
\mathcal{A} x^{p-1} y^{q}=\lambda x^{[l-1]} \\
\mathcal{A} x^{p} y^{q-1}=\lambda y^{[l-1]}
\end{array}\right.
$$

where $x^{[\alpha]}=\left[x_{1}^{\alpha}, \ldots, x_{n}^{\alpha}\right]^{T}$ and $l=p+q$, then $\lambda$ is called the singular value of $\mathcal{A}$, and $(x, y)$ is the left and right eigenvector pair of $\mathcal{A}$, associated with $\lambda$. If $\lambda \in \mathbb{R}, x \in \mathbb{R}^{m}$, and $y \in \mathbb{R}^{n}$, then we say that $\lambda$ is an $H$-singular value of $\mathcal{A}$, and $(x, y)$ is the left and right $H$-eigenvector pair associated with $\lambda$; see [1-4].

We call $\mathcal{A}$ an elasticity tensor, if $p=q=2, m=n=2$ or 3 , and $\mathcal{A}$ is a real partially symmetric rectangular tensor. When $\mathcal{A}$ is an elasticity tensor, the strong ellipticity condition holds if and only if $\mathcal{A}$ is positive definite, the strong ellipticity condition plays an important role in the theory of elasticity $[5,6]$. By introducing the $\mathrm{H}$-singular values of a partially symmetric tensor, Chang et al. [1] provided a necessary and sufficient condition for the positive definiteness of a partially symmetric rectangular tensor as follows.

Theorem 1 ([1]) Suppose that $\mathcal{A} \in \mathbb{R}^{[p ; q ; m ; n]}$ is a partially symmetric rectangular tensor, $p$ and $q$ are even. Then $\mathcal{A}$ is positive definite, i.e.,

$$
f(x, y)=\mathcal{A} x^{p} y^{q}=\sum_{i_{1}, \ldots, i_{p}=1}^{m} \sum_{j_{1}, \ldots, j_{q}=1}^{n} a_{i_{1} \ldots i_{p} j_{1} \ldots j_{q}} x_{i_{1}} \cdots x_{i_{p}} y_{j_{1}} \cdots y_{j_{q}}>0
$$

for all nonzero vectors $x \in \mathbb{R}^{m}, y \in \mathbb{R}^{n}$ if and only if all of its $H$-singular values are positive.

In 2005, Qi obtained a Geršgorin-type H-eigenvalue localization set for real symmetric tensors, which is useful in verifying the positive definiteness of square tensor $\mathcal{A}[7,8]$. And then some improved results are given in [9-12]. Recently, the problem of singular value inclusion sets for rectangular tensors became an important topic [13-15]. Unfortunately, these singular value inclusion sets of rectangular tensors cannot be used to verify the positive definiteness of a rectangular tensor.

Inspired by the above, in Sect. 2, we intend to introduce the definition of $\mathrm{V}$-singular value for a rectangular tensor and study its properties. In Sect. 3, we give some V-singular value inclusion sets for rectangular tensors with positive diagonal entries. As applications, we give an upper bound for the largest $\mathrm{V}$-singular value of nonnegative rectangular tensors in Sect. 4, and sufficient conditions of the positive definiteness for rectangular tensors are also provided in Sect. 5 .

\section{V-singular values of rectangular tensors}

First, we introduce the definition of a V-singular value for a rectangular tensor, which can be viewed as a variant of the definition of $\mathrm{H}$-singular value $[1,2]$. 
Definition 1 Let $\mathcal{A} \in \mathbb{R}^{[p ; ; ; m ; n]}, p, q \geq 2$. If there exist a number $\lambda \in \mathbb{R}$, vectors $x \in \mathbb{R}^{m} \backslash\{0\}$, and $y \in \mathbb{R}^{n} \backslash\{0\}$ such that

$$
\left\{\begin{array}{l}
\mathcal{A} x^{p-1} y^{q}=\lambda x^{[p-1]}, \\
\mathcal{A} x^{p} y^{q-1}=\lambda y^{[q-1]}, \\
\sum_{i=1}^{m} x_{i}^{p}=1, \\
\sum_{j=1}^{n} y_{j}^{q}=1,
\end{array}\right.
$$

then $\lambda$ is called the $V$-singular value of $\mathcal{A}$, and $(x, y)$ is the left and right eigenvector pair of $\mathcal{A}$ associated with $\lambda$.

Remark 1 When $p=2, q=2$, our definition is the same as the M-eigenvalue which is introduced in [16].

Remark 2 When $p$ is even and $q$ is even, our definition can be viewed as a special case of the $l^{k, s}$-singular value which is introduced in [4]. However, when one of $p, q$ is odd, the definition of V-singular value is different from the $l^{k, s}$-singular value.

Theorem 2 Let $\mathcal{A} \in \mathbb{R}^{[p ; q ; m ; n]}$. We have the following conclusions:

(a) If $x$ and $y$ are left and right eigenvectors of $\mathcal{A}$, associated with a V-singular value $\lambda$ of $\mathcal{A}$, then

$$
\lambda=f(x, y)=\mathcal{A} x^{p} y^{q} .
$$

(b) When $p$ is odd and $q$ is odd, then $(\lambda,-x,-y),(-\lambda,-x, y)$, and $(-\lambda, x,-y)$ are also $V$-singular values and their associated eigenvectors of $\mathcal{A}$.

(c) When $p$ is odd and $q$ is even, then $(\lambda, x,-y),(-\lambda,-x, y)$, and $(-\lambda,-x,-y)$ are also $V$-singular values and their associated eigenvectors of $\mathcal{A}$.

(d) When $p$ is even and $q$ is even, then $(\lambda,-x,-y),(\lambda,-x, y)$, and $(\lambda, x,-y)$ are also $V$-singular values and their associated eigenvectors of $\mathcal{A}$.

Proof By the first equation of (3), we have

$$
f(x, y)=\mathcal{A} x^{p} y^{q}=\lambda\left(x^{p-1}\right)^{T} x=\lambda \sum_{i=1}^{m} x_{i}^{p}=\lambda .
$$

By the second equation of (3), we have

$$
f(x, y)=\mathcal{A} x^{p} y^{q}=\lambda\left(y^{q-1}\right)^{T} y=\lambda \sum_{j=1}^{n} y_{j}^{q}=\lambda .
$$

We thus have conclusion (a).

It is straightforward to verify the assertions (b), (c) and (d). 


\section{V-singular value inclusion sets for rectangular tensors with positive diagonal} entries

Let $\sigma(\mathcal{A})$ be the set containing all V-singular values of $\mathcal{A}=\left(a_{i_{1} \ldots i_{p} j_{1} \ldots j_{q}}\right) \in \mathbb{R}^{[p ; q ; m ; n]}$. We call the entries $a_{i \ldots . . i j . . . j}(i \in[m], j \in[n])$ diagonal, and other entries are called off-diagonal. In this section, some new $\mathrm{V}$-singular value inclusion sets are given to locate all $\mathrm{V}$-singular values of rectangular tensors with positive diagonal entries.

Theorem 3 Consider $\mathcal{A}=\left(a_{i_{1} \ldots i_{p} j_{1} \ldots j_{q}}\right) \in \mathbb{R}^{[p ; q ; m ; n]}$ with positive diagonal entries, $p$ and $q$ even, and $\Delta=\left\{\left(j_{1}, \ldots, j_{q}\right):(1, \ldots, 1), \ldots,(n, \ldots, n)\right\}$. Then

$$
\sigma(\mathcal{A}) \subseteq \Gamma_{1}(\mathcal{A})=\bigcup_{i \in[m]}\left\{z \in \mathbb{R}:\left|z-\underline{\alpha_{i}}\right| \leq R_{i}(\mathcal{A})+\overline{\alpha_{i}}-\underline{\alpha_{i}}\right\}
$$

where $\min _{i \in[m]}\left\{a_{i \ldots i 1 \ldots 1}, \ldots, a_{i \ldots i n \ldots . . .}\right\}=\underline{\alpha_{i}}, \max _{i \in[m]}\left\{a_{i \ldots i 1 \ldots 1}, \ldots, a_{i \ldots i n . . . n}\right\}=\overline{\alpha_{i}}$,

$$
\begin{aligned}
& R_{i}(\mathcal{A})=\sum_{i_{2}, \ldots, i_{p}=1}^{m} \sum_{\substack{\left.j_{1}, \ldots, j_{q}\right)=1,\left(j_{1}, \ldots, j_{q}\right) \notin \Delta}}^{n}\left|a_{i i_{2} \ldots i_{p} j_{1} \ldots j_{q}}\right|+\gamma_{i}, \\
& \gamma_{i}=\max _{k \in[n]}\left\{\sum_{\substack{i_{2}, \ldots, i_{p}=1,\left(i_{2}, \ldots, i_{p}\right) \neq(i, \ldots, i)}}^{m}\left|a_{i i_{2} \ldots i_{p} k \ldots k}\right|\right\} .
\end{aligned}
$$

Proof Assume that $\lambda$ is a V-singular value of $\mathcal{A}, x=\left(x_{i}\right)_{i=1}^{m} \in \mathbb{R}^{m} \backslash\{0\}$ and $y=\left(y_{j}\right)_{j=1}^{n} \in$ $\mathbb{R}^{n} \backslash\{0\}$ are the corresponding left and right eigenvectors. Let $\left|x_{s}\right|=\max _{i \in[m]}\left\{\left|x_{i}\right|\right\}$. Then $\left|x_{s}\right| \neq 0$. The $s$ th equation of $\mathcal{A} x^{p-1} y^{q}=\lambda x^{[p-1]}$ is

$$
\begin{aligned}
& \lambda x_{s}^{p-1}=\sum_{i_{2}, \ldots, i_{p}=1}^{m} \sum_{j_{1}, \ldots, j_{q}=1}^{n} a_{s i_{2} \ldots i_{p} j_{1} \ldots j_{q}} x_{i_{2}} \cdots x_{i_{p}} y_{j_{1}} \cdots y_{j_{q}} \\
& =\sum_{\substack{i_{2}, \ldots, i_{p}=1,\left(i_{2}, \ldots, i_{p}\right)(s, \ldots, s)}}^{m} \sum_{\substack{j_{1}, \ldots, j_{q}=1,\left(j_{1}, \ldots, j_{q}\right) \in \Delta}}^{n} a_{s i_{2} \ldots i_{p} j_{1} \ldots j_{q}} x_{i_{2}} \cdots x_{i_{p}} y_{j_{1}} \cdots y_{j_{q}} \\
& +\sum_{\substack{i_{2}, \ldots, i_{p}=1,\left(i_{2}, \ldots, i_{p}\right)=(s, \ldots, s)}}^{m} \sum_{\substack{j_{1}, \ldots, j_{q}=1,\left(j_{1}, \ldots, j_{q}\right) \notin \Delta}}^{n} a_{s i_{2} \ldots i_{p} j_{1} \ldots . . j_{q}} x_{i_{2}} \cdots x_{i_{p}} y_{j_{1}} \cdots y_{j_{q}} \\
& +\sum_{\substack{i_{2}, \ldots, i_{p}=1,\left(i_{2}, \ldots, i_{p}\right) \neq(s, \ldots, s)}}^{m} \sum_{\substack{j_{1}, \ldots, j_{q}=1,\left(j_{1}, \ldots, j_{q}\right) \notin \Delta}}^{n} a_{s i_{2} \ldots i_{p} j_{1} \ldots j_{q}} x_{i_{2}} \cdots x_{i_{p}} y_{j_{1}} \cdots y_{j_{q}} \\
& +\sum_{\substack{i_{2}, \ldots, i_{p}=1, i_{2}, \ldots, i_{p} \neq s_{s}, \ldots, j_{1}}}^{m} \sum_{\substack{\left.j_{1}, \ldots, \ldots, j_{q}\right) \in \Delta \\
j_{q}=1,}}^{n} a_{s i_{2} \ldots i_{p} j_{1} \ldots j_{q}} x_{i_{2}} \cdots x_{i_{p}} y_{j_{1}} \cdots y_{j_{q}},
\end{aligned}
$$

which can be rewritten as

$$
\left(\lambda-\left(a_{s . . . s 1 \ldots 1} y_{1}^{q}+\cdots+a_{s \ldots . . . n n} \ldots y_{n}^{q}\right)\right) x_{s}^{p-1}
$$




$$
\begin{aligned}
& =\sum_{\substack{i_{2}, \ldots, i_{p}=1,\left(i_{2}, \ldots, i_{p}\right)=(s, \ldots, s)}}^{m} \sum_{\substack{j_{1}, \ldots, j_{q}=1,\left(j_{1}, \ldots, j_{q}\right) \notin \Delta}}^{n} a_{s i_{2} \ldots i_{p} j_{1} \ldots j_{q}} x_{i_{2}} \cdots x_{i_{p}} y_{j_{1}} \cdots y_{j_{q}} \\
& +\sum_{\substack{i_{2}, \ldots, i_{p}=1,\left(i_{2}, \ldots, i_{p}\right) \neq(s, \ldots, s)}}^{m} \sum_{\substack{j_{1}, \ldots, j_{q}=1,\left(j_{1}, \ldots, j_{q}\right) \notin \Delta}}^{n} a_{s i_{2} \ldots i_{p} j_{1} \ldots j_{q}} x_{i_{2}} \cdots x_{i_{p}} y_{j_{1}} \cdots y_{j_{q}} \\
& +\sum_{\substack{i_{2}, \ldots, i_{p}=1, i_{2}, \ldots, i_{p} \neq s, \ldots, s,}}^{m} \sum_{j_{1}, \ldots, j_{q}=1,}^{n} a_{\left.s i_{1}, \ldots, j_{q}\right) \in \Delta}
\end{aligned}
$$

Therefore,

$$
\begin{aligned}
(\lambda & \left.-\underline{\alpha_{s}}\left(y_{1}^{q}+\cdots+y_{n}^{q}\right)\right) x_{s}^{p-1} \\
= & \sum_{\substack{i_{2}, \ldots, i_{p}=1,\left(i_{2}, \ldots, i_{p}\right)=(s, \ldots, s)}}^{m} \sum_{\substack{j_{1}, \ldots, j_{q}=1,\left(j_{1}, \ldots, j_{q}\right) \notin \Delta}}^{n} a_{s i_{2} \ldots i_{p} j_{1} \ldots j_{q}} x_{i_{2}} \cdots x_{i_{p}} y_{j_{1}} \cdots y_{j_{q}} \\
& +\sum_{\substack{i_{2}, \ldots, i_{p}=1,\left(i_{2}, \ldots, i_{p}\right) \neq(s, \ldots, s)}}^{m} \sum_{\substack{j_{1}, \ldots, j_{q}=1,\left(j_{1}, \ldots, j_{q}\right) \notin \Delta}}^{n} a_{s i_{2} \ldots i_{p} j_{1} \ldots j_{q}} x_{i_{2}} \cdots x_{i_{p}} y_{j_{1}} \cdots y_{j_{q}} \\
& +\sum_{\substack{i_{2}, \ldots, i_{p}=1,\left(i_{2}, \ldots, i_{p}\right) \neq(s, \ldots, s)}}^{m} \sum_{\substack{j_{1}, \ldots, j_{q}=1,\left(j_{1}, \ldots, j_{q}\right) \in \Delta}}^{n} a_{s i_{2} \ldots i_{p} j_{1} \ldots j_{q}} x_{i_{2}} \cdots x_{i_{p}} y_{j_{1}} \cdots y_{j_{q}} \\
& +\left(a_{s . . . s 1 \ldots 1}-\underline{\left.\alpha_{s}\right)} x_{s}^{p-1} y_{1}^{q}+\cdots+\left(a_{s . . . s n n . . n}-\underline{\alpha_{s}}\right) x_{s}^{p-1} y_{n}^{q} .\right.
\end{aligned}
$$

By taking absolute values on both sides and the fact that $\sum_{j=1}^{n} y_{j}^{q}=1, q$ is even, we have

$$
\begin{aligned}
& \left|\lambda-\underline{\alpha_{s}}\right|\left|x_{s}^{p-1}\right| \leq \sum_{\substack{i_{2}, \ldots, i_{p}=1,\left(i_{2}, \ldots, i_{p}\right)=(s, \ldots, s)}}^{m} \sum_{\substack{j_{1}, \ldots, j_{q}=1,\left(j_{1}, \ldots, j_{q}\right) \notin \Delta}}^{n}\left|a_{s i_{2} \ldots i_{p} j_{1} \ldots j_{q}}\right|\left|x_{i_{2}}\right| \cdots\left|x_{i_{p}}\right|\left|y_{j_{1}}\right| \cdots\left|y_{j_{q}}\right| \\
& +\sum_{\substack{i_{2}, \ldots, i_{p}=1,\left(i_{2}, \ldots, i_{p}\right) \neq(s, \ldots, s)}}^{m} \sum_{\substack{j_{1}, \ldots, j_{q}=1,\left(j_{1}, \ldots, j_{q}\right) \notin \Delta}}^{n}\left|a_{s i_{2} \ldots i_{p} j_{1} \ldots . . j_{q}}\right|\left|x_{i_{2}}\right| \cdots\left|x_{i_{p}}\right|\left|y_{j_{1}}\right| \cdots\left|y_{j_{q}}\right| \\
& +\sum_{\substack{i_{2}, \ldots, i_{p}=1,\left(i_{2}, \ldots, i_{p}\right) \neq(s, \ldots, s)}}^{m} \sum_{\substack{j_{1}, \ldots, j_{q}=1,\left(j_{1}, \ldots, j_{q}\right) \in \Delta}}^{n}\left|a_{s i_{2} \ldots i_{p} j_{1} \ldots j_{q}}\right|\left|x_{i_{2}}\right| \cdots\left|x_{i_{p}}\right|\left|y_{j_{1}}\right| \cdots\left|y_{j_{q}}\right| \\
& +\left|a_{s . . . s 1 \ldots 1}-\underline{\alpha_{s}}\right|\left|x_{s}^{p-1}\right|\left|y_{1}^{q}\right|+\cdots+\left|a_{s \ldots s n \ldots n}-\underline{\alpha_{s}}\right|\left|x_{s}^{p-1}\right|\left|y_{n}^{q}\right| \\
& \leq \sum_{\substack{i_{2}, \ldots, i_{p}=1,\left(i_{2}, \ldots, i_{p}\right)=(s, \ldots, s)}}^{m} \sum_{\substack{j_{1}, \ldots, j_{q}=1,\left(j_{1}, \ldots, j_{q}\right) \notin \Delta}}^{n}\left|a_{s i_{2} \ldots i_{p} j_{1} \ldots j_{q}}\right|\left|x_{i_{2}}\right| \cdots\left|x_{i_{p}}\right|\left|y_{j_{1}}\right| \cdots\left|y_{j_{q}}\right| \\
& +\sum_{\substack{i_{2}, \ldots, i_{p}=1,\left(i_{2}, \ldots, i_{p}\right) \neq(s, \ldots, s)}}^{m} \sum_{\substack{j_{1}, \ldots, j_{q}=1,\left(j_{1}, \ldots, j_{q}\right) \notin \Delta}}^{n}\left|a_{s i_{2} \ldots i_{p} j_{1} \ldots j_{q}}\right|\left|x_{i_{2}}\right| \cdots\left|x_{i_{p}}\right|\left|y_{j_{1}}\right| \cdots\left|y_{j_{q}}\right|
\end{aligned}
$$




$$
\begin{aligned}
& +\sum_{\substack{i_{2}, \ldots, i_{p}=1,\left(i_{2}, \ldots, i_{p}\right) \neq(s, \ldots, s)}}^{m} \sum_{\substack{j_{1}, \ldots, j_{q}=1,\left(j_{1}, \ldots, j_{q}\right) \in \Delta}}^{n}\left|a_{s i_{2} \ldots i_{p} j_{1} \ldots j_{q}}\right|\left|x_{i_{2}}\right| \cdots\left|x_{i_{p}}\right|\left|y_{j_{1}}\right| \cdots\left|y_{j_{q}}\right| \\
& +\left(\overline{\alpha_{s}}-\underline{\alpha_{s}}\right)\left(y_{1}^{q}+\cdots+y_{n}^{q}\right)\left|x_{s}^{p-1}\right| .
\end{aligned}
$$

Note that

$$
\begin{aligned}
& \sum_{\substack{i_{2}, \ldots, i_{p}=1,\left(i_{2}, \ldots, i_{p}\right) \neq(s, \ldots, s)}}^{m} \sum_{\substack{j_{1}, \ldots, j_{q}=1,\left(j_{1}, \ldots, j_{q}\right) \in \Delta}}^{n}\left|a_{s i_{2} \ldots i_{p} j_{1} \ldots j_{q}}\right|\left|x_{i_{2}}\right| \cdots\left|x_{i_{p}}\right|\left|y_{j_{1}}\right| \cdots\left|y_{j_{q}}\right| \\
\leq & \left(\sum_{\substack{i_{2}, \ldots, i_{p}=1,\left(i_{2}, \ldots, i_{p}\right) \neq(s, \ldots, s)}}^{m}\left|a_{s i_{2} \ldots i_{p} 1 \ldots 1}\right|\left|y_{1}^{q}\right|+\cdots+\sum_{\substack{i_{2}, \ldots, i_{p}=1,\left(i_{2}, \ldots, i_{p}\right) \neq(s, \ldots, s)}}^{m}\left|a_{s i_{2} \ldots i_{p} n \ldots . . n}\right|\left|y_{n}^{q}\right|\right)\left|x_{s}^{p-1}\right| \\
\leq & \max _{k \in[n]}\left\{\sum_{\substack{\left(i_{2}, \ldots, i_{p}\right) \neq(s, \ldots, s) \\
i_{2}, \ldots, i_{p}=1,}}^{m}\left|a_{s i_{2} \ldots i_{p} k \ldots k}\right|\right\}\left(y_{1}^{q}+\cdots+y_{n}^{q}\right)\left|x_{s}^{p-1}\right| \\
= & \max _{k \in[n]}\left\{\sum_{\substack{i_{2}, \ldots, i_{p}=1,\left(i_{2}, \ldots, i_{p}\right) \neq(s, \ldots, s)}}^{m}\left|a_{s i_{2} \ldots i_{p} k \ldots k}\right|\right\}\left|x_{s}^{p-1}\right| .
\end{aligned}
$$

Then,

$$
\left|\lambda-\underline{\alpha_{s}}\right|\left|x_{s}^{p-1}\right| \leq R_{s}(\mathcal{A})\left|x_{s}^{p-1}\right|+\left(\overline{\alpha_{s}}-\underline{\alpha_{s}}\right)\left|x_{s}^{p-1}\right| .
$$

Dividing $\left|x_{s}\right|$ on both sides of (4) yields

$$
\left|\lambda-\underline{\alpha_{s}}\right| \leq R_{s}(\mathcal{A})+\left(\overline{\alpha_{s}}-\underline{\alpha_{s}}\right) .
$$

Thus $\sigma(\mathcal{A}) \subseteq \Gamma_{1}(\mathcal{A})$. This completes the proof.

Similarly, using the forms $\mathcal{A} x^{p} y^{q-1}=\lambda y^{[q-1]}$ and $\sum_{i=1}^{m} x_{i}^{p}=1$, we can get the following V-singular value interval.

Theorem 4 Consider $\mathcal{A}=\left(a_{i_{1} \ldots i_{p} j_{1} \ldots j_{q}}\right) \in \mathbb{R}^{[p ; q ; m ; n]}$ with positive diagonal entries, $p$ and $q$ even, and $\Omega=\left\{\left(i_{1}, \ldots, i_{p}\right):(1, \ldots, 1), \ldots,(m, \ldots, m)\right\}$. Then

$$
\sigma(\mathcal{A}) \subseteq \Gamma_{2}(\mathcal{A})=\bigcup_{j \in[n]}\left\{z \in \mathbb{R}:\left|z-\underline{\beta_{j}}\right| \leq C_{j}(\mathcal{A})+\overline{\beta_{j}}-\underline{\beta_{j}}\right\}
$$

where $\min _{j \in[n]}\left\{a_{1 \ldots 1 j \ldots j}, \ldots, a_{m \ldots m j \ldots j}\right\}=\underline{\beta_{j}}, \max _{j \in[n]}\left\{a_{1 \ldots 1 j \ldots j}, \ldots, a_{m \ldots m j \ldots j}\right\}=\overline{\beta_{j}}$,

$$
\begin{aligned}
& C_{j}(\mathcal{A})=\sum_{\substack{i_{1}, \ldots, i_{p}=1, j_{2}, \ldots, j_{q}=1 \\
\left(i_{1}, \ldots, i_{p}\right) \notin \Omega}}^{m}\left|a_{i_{1} \ldots i_{p} j j_{2} \ldots j_{q}}\right|+\delta_{j}, \\
& \delta_{j}=\max _{k \in[m]}\left\{\sum_{\substack{j_{2}, \ldots, j_{q}=1,\left(j_{2}, \ldots, j_{q}\right) \neq(j, \ldots, j)}}^{n}\left|a_{k \ldots k j j_{2} \ldots j_{q}}\right|\right\} .
\end{aligned}
$$




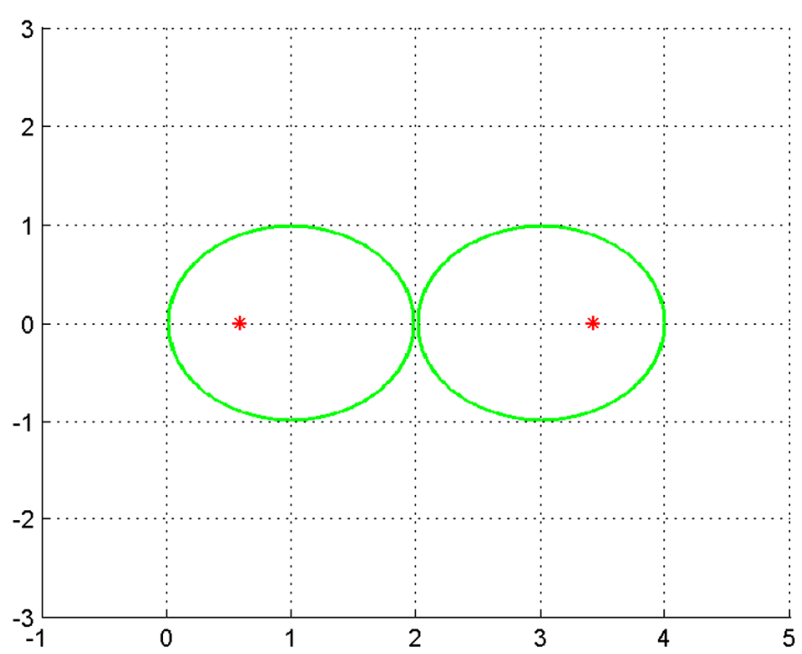

Figure 1 The V-singular value inclusion set $\Gamma(\mathcal{A})$ and the exact V-singular values

By Theorems 3 and 4, we can get an inclusion set to locate all V-singular value of a rectangular tensor.

Corollary 1 Consider $\mathcal{A}=\left(a_{i_{1} \ldots i_{p} j_{1} \ldots j_{q}}\right) \in \mathbb{R}^{[p ; ; ; m ; n]}$ with positive diagonal entries, as well as $p$ and $q$ even. Then

$$
\sigma(\mathcal{A}) \subseteq \Gamma(\mathcal{A})=\Gamma_{1}(\mathcal{A}) \cap \Gamma_{2}(\mathcal{A})
$$

Example 1 Let us consider the rectangular tensor $\mathcal{A} \in \mathbb{R}^{[2 ; 4 ; 2 ; 2]}$ with

$$
\begin{aligned}
& a_{111111}=a_{112222}=1, \quad a_{221111}=a_{222222}=3, \\
& a_{121111}=a_{122222}=a_{211111}=a_{212222}=-1,
\end{aligned}
$$

and other $a_{i_{1} i_{2} j_{1} \ldots j_{4}}=0$.

The $\mathrm{V}$-singular value inclusion set $\Gamma(\mathcal{A})$ and the exact $\mathrm{V}$-singular values are drawn in Fig. 1, where $\Gamma(\mathcal{A})$ is represented by solid boundary, and the exact $\mathrm{V}$-singular values are plotted by "*:" It is easy to see that $\sigma(\mathcal{A})=\{2 \pm \sqrt{2}\} \subseteq \Gamma(\mathcal{A})$, that is, $\Gamma(\mathcal{A})$ can capture all $\mathrm{V}$-singular values of $\mathcal{A}$.

\section{Nonnegative rectangular tensors}

In this section, we give some results for the $\mathrm{V}$-singular value of nonnegative rectangular tensors. The following definition for irreducibility has been introduced in [17].

Definition 2 The square tensor $\mathcal{A}$ is called reducible if there exists a nonempty proper index subset $\mathbb{J} \subset\{1,2, \ldots, n\}$ such that $a_{i_{1}, i_{2}, \ldots, i_{m}}=0, \forall i_{1} \in \mathbb{J}, \forall i_{2}, \ldots, i_{m} \notin \mathbb{J}$. If $\mathcal{A}$ is not reducible, then we call $\mathcal{A}$ irreducible.

The Perron-Frobenius theorem for nonnegative square tensors is introduced in [18], which states that the spectral radius of any nonnegative square tensor is an eigenvalue with a nonnegative eigenvector, and the eigenvector is positive and unique if the square 
tensor is irreducible. For any $j \in[n]$, let $\mathcal{A}(\cdot, j)=\left(a_{i_{1} \ldots j_{j} \ldots . . j}\right)$ be a $p$ th order $m$-dimensional square tensor. For any $i \in[m]$, let $\mathcal{A}(\cdot, i)=\left(a_{i \ldots . . i_{1} \ldots j_{q}}\right)$ be a $q$ th order $n$-dimensional square tensor.

Definition 3 ([1]) A nonnegative rectangular tensor $\mathcal{A}$ is called irreducible if all the square tensors $\mathcal{A}(\cdot, j), j \in[n]$, and $\mathcal{A}(\cdot, i), i \in[m]$, are irreducible.

Denote by $\lambda_{\max }(\mathcal{A})$ the maximal $V$-singular value of $\mathcal{A} \in \mathbb{R}^{[p ; q ; m ; n]}$. Then

$$
\lambda_{\max }(\mathcal{A})=\max \left\{\mathcal{A} x^{p} y^{q}, \sum_{i=1}^{m} x_{i}^{p}=1, \sum_{j=1}^{n} y_{j}^{q}=1\right\} .
$$

And we call

$$
\rho_{V}(\mathcal{A})=\max \{|\lambda|: \lambda \in \sigma(\mathcal{A})\}
$$

the largest $\mathrm{V}$-singular value of $\mathcal{A}$. We have the following result.

Theorem 5 Let $\mathcal{A}=\left(a_{i_{1} \ldots i_{p} j_{1} \ldots j_{q}}\right) \in \mathbb{R}_{+}^{[p ; q ; m ; n]}$. Then, $\rho_{V}(\mathcal{A})=\lambda_{\max }(\mathcal{A})$, and there is a pair of nonnegative eigenvectors corresponding to $\rho_{V}(\mathcal{A})$. Furthermore, if $\mathcal{A}$ is irreducible, then $\rho_{V}(\mathcal{A})=\lambda_{\max }(\mathcal{A})$, and there is a pair of positive eigenvectors corresponding to $\rho_{V}(\mathcal{A})$.

Proof Assume that $\lambda$ is a V-singular value of $\mathcal{A}, x=\left(x_{i}\right)_{i=1}^{m} \in \mathbb{R}^{m}$ and $y=\left(y_{j}\right)_{j=1}^{n} \in \mathbb{R}^{n}$ are the corresponding left and right eigenvectors. Then $\lambda=\mathcal{A} x^{p} y^{q}$ and, by the nonnegativity of the entries of $\mathcal{A}$, we have

$$
|\lambda| \leq \lambda_{\max }(\mathcal{A})
$$

Next, we consider the eigenvectors of $\rho_{V}(\mathcal{A})$. Assume that $\lambda_{\max }(\mathcal{A})$ is the maximal Vsingular value of $\mathcal{A}, \mu$ and $v$ are the corresponding left and right eigenvectors, and one of $\mu$ or $v$ is not a nonnegative vector. Then we have

$$
\lambda_{\max }(\mathcal{A})=\mathcal{A} \mu^{p} v^{q} \leq \mathcal{A}\left|\mu^{p}\right|\left|\nu^{q}\right| \leq \lambda_{\max }(\mathcal{A}),
$$

which implies $\lambda_{\max }(\mathcal{A})=\mathcal{A}\left|\mu^{p}\right|\left|v^{q}\right|$. Therefore, $|\mu|$ and $|\nu|$ are the left and right nonnegative eigenvectors corresponding to $\lambda_{\max }(\mathcal{A})$.

If $\mathcal{A}$ is irreducible, by Lemma 2 in [1], $\mathcal{A}\left|\mu^{p-1}\right|\left|\nu^{q}\right| \neq 0, \mathcal{A}\left|\mu^{p}\right|\left|\nu^{q-1}\right| \neq 0$, that is to say, $|\mu|>0$ and $|\nu|>0$.

Hence, the proof is completed.

From Theorem 5, we obtain the the following corollary.

Corollary 2 Let $\mathcal{A} \in \mathbb{R}_{+}^{[p ; q ; m ; n]}$. Then,

$$
\rho_{V}(\mathcal{A})=\max \left\{\mathcal{A} x^{p} y^{q}, \sum_{i=1}^{m} x_{i}^{p}=1, \sum_{j=1}^{n} y_{j}^{q}=1\right\} .
$$

We have the following lemma. 
Lemma 1 Let $\mathcal{A} \in \mathbb{R}_{+}^{[p ; q ; m ; n]}$. Then,

$$
\rho_{V}(\mathcal{A}) \geq \max _{i \in[m], j \in[n]}\left\{a_{i \ldots . . j \ldots . . . j}\right\}
$$

Proof Let $e_{i}$ be an $m$-dimensional real vector, whose $i$ th entry is 1 , and others are 0 , also let $e_{j}$ be an $n$-dimensional real vector, whose $j$ th entry is 1 and others are 0 . Hence, from Corollary 2,

$$
\rho(\mathcal{A}) \geq \mathcal{A} e_{i} \cdots e_{i} e_{j} \cdots e_{j}=a_{i \ldots i j . . . j}
$$

for any $i \in[m], j \in[n]$. This means $\rho(\mathcal{A}) \geq \max _{i \in[m], j \in[n]}\left\{a_{i \ldots i j \ldots . . j}\right\}$.

Based on Theorem 5, an upper bound for the largest V-singular value of nonnegative rectangular tensors is given.

Theorem 6 Let $\mathcal{A} \in \mathbb{R}^{[p ; q ; m ; n]}$ be nonnegative. Then,

$$
\rho_{V}(\mathcal{A}) \leq \min \left\{\max _{i \in[m]}\left\{R_{i}(\mathcal{A})+\overline{\alpha_{i}}\right\}, \max _{j \in[n]}\left\{C_{j}(\mathcal{A})+\overline{\beta_{j}}\right\}\right\} .
$$

Proof Because $\rho_{V}(\mathcal{A})$ is a V-singular value of $\mathcal{A}, x=\left(x_{i}\right)_{i=1}^{m} \in \mathbb{R}^{m} \backslash\{0\}$ and $y=\left(y_{j}\right)_{j=1}^{n} \in$ $\mathbb{R}^{n} \backslash\{0\}$ are the corresponding left and right eigenvectors. From Theorem 5, we have $x, y \geq 0$. Let $x_{s}=\max _{i \in[m]}\left\{x_{i}\right\}$. Then $x_{s}>0$. The sth equation of $\mathcal{A} x^{p-1} y^{q}=\lambda x^{[p-1]}$ is

$$
\begin{aligned}
& \rho_{V}(\mathcal{A}) x_{s}^{p-1}=\sum_{i_{2}, \ldots, i_{p}=1}^{m} \sum_{j_{1}, \ldots, j_{q}=1}^{n} a_{s i_{2} \ldots i_{p} j_{1} \ldots j_{q}} x_{i_{2}} \cdots x_{i_{p}} y_{j_{1}} \cdots y_{j_{q}} \\
& =\sum_{\substack{i_{2}, \ldots, i_{p}=1,\left(i_{2}, \ldots, i_{p}\right)=(s, \ldots, s)}}^{m} \sum_{\substack{j_{1}, \ldots, j_{q}=1,\left(j_{1}, \ldots, j_{q}\right) \in \Delta}}^{n} a_{s i_{2} \ldots i_{p} j_{1} \ldots j_{q}} x_{i_{2}} \cdots x_{i_{p}} y_{j_{1}} \cdots y_{j_{q}} \\
& +\sum_{\substack{i_{2}, \ldots, i_{p}=1,\left(i_{2}, \ldots, i_{p}\right)\left(s_{1}, \ldots, s\right)}}^{m} \sum_{\substack{j_{1}, \ldots, j_{j}=1,\left(j_{1}, \ldots, j_{q}\right) \in \Delta}}^{n} a_{s i_{2} \ldots i_{p} j_{1} \ldots j_{q}} x_{i_{2}} \cdots x_{i_{p}} y_{j_{1}} \cdots y_{j_{q}} \\
& +\sum_{i_{2}, \ldots, i_{p}=1}^{m} \sum_{\substack{j_{1}, \ldots, j_{q}=1,\left(j_{1}, \ldots, j_{q} \notin \notin \Delta\right.}}^{n} a_{s i_{2} \ldots i_{p} j_{1} \ldots j_{q}} x_{i_{2}} \cdots x_{i_{p}} y_{j_{1}} \cdots y_{j_{q}} \\
& \leq R_{s}(\mathcal{A}) x_{s}^{p-1}+\overline{\alpha_{s}} x_{s}^{p-1} .
\end{aligned}
$$

Then,

$$
\rho_{V}(\mathcal{A}) \leq R_{s}(\mathcal{A})+\overline{\alpha_{s}}
$$

Similarly, let $y_{t}=\max _{j \in[n]}\left\{y_{j}\right\}$. Then $y_{t}>0$. Considering the $t$ th equation of $\mathcal{A} x^{p} y^{q-1}=$ $\lambda y^{[q-1]}$, we can get

$$
\rho_{V}(\mathcal{A}) \leq C_{t}(\mathcal{A})+\overline{\beta_{t}} .
$$

The proof is completed. 
Example 2 Let us consider the rectangular tensor $\mathcal{A} \in \mathbb{R}^{[2 ; 4 ; 2 ; 2]}$ with

$$
\begin{aligned}
& a_{111111}=a_{112222}=3, \quad a_{221111}=a_{222222}=3, \\
& a_{121111}=a_{122222}=a_{211111}=a_{212222}=1,
\end{aligned}
$$

and other $a_{i_{1} i_{2} j_{1} \ldots j_{4}}=0$.

By Theorem 6, we have

$$
\rho_{V}(\mathcal{A}) \leq 4
$$

In fact, $\rho_{V}(\mathcal{A})=4$.

Example 3 Let us consider the rectangular tensor $\mathcal{A} \in \mathbb{R}^{[3 ; 2 ; 2 ; 2]}$ with

$$
\begin{array}{ll}
a_{11111}=a_{11122}=3, & a_{22211}=a_{22222}=3, \\
a_{11211}=a_{11222}=1, & a_{21111}=a_{21122}=1,
\end{array}
$$

and other $a_{i_{1} i_{2} i_{3} j_{1} j_{2}}=0$.

By Theorem 6, we have

$$
\rho_{V}(\mathcal{A}) \leq 4
$$

In fact, $\rho_{V}(\mathcal{A})=4$.

\section{Positive definiteness of rectangular tensors}

In this section, we provide some verifiable sufficient conditions for the positive definiteness of rectangular tensors. We have the following theorem.

Theorem 7 Suppose that $\mathcal{A} \in \mathbb{R}^{[p ; q ; m ; n]}$ is a partially symmetric rectangular tensor, $p$ and $q$ are even. Then,

(a) There exist $V$-singular value of $\mathcal{A}$ and associated left and right eigenvectors.

(b) $\mathcal{A}$ is positive definite if and only if all of its $V$-singular values are positive.

Proof Consider the following optimization problem:

$$
\min \left\{f(x, y)=\mathcal{A} x^{p} y^{q}: \sum_{i=1}^{m} x_{i}^{p}=1, \sum_{j=1}^{n} y_{j}^{q}=1\right\} .
$$

The objective function $f(x, y)$ is continuous in variables $x$ and $y$. When $p$ and $q$ are even, the feasible set $\left\{(x, y) \in \mathbb{R}^{m} \times \mathbb{R}^{n}: \sum_{i=1}^{m} x_{i}^{p}=1, \sum_{j=1}^{n} y_{j}^{q}=1\right\}$ is compact. Then, there exist vectors $x_{*}$ and $y_{*}$ that solve the optimization problem with the minimal objective value $\lambda_{*}=\mathcal{A} x_{*}^{p} y_{*}^{q}$. 
By the Lagrangian multiplier method, for the optimal solution $\left(x_{*}, y_{*}\right)$, there exist multipliers $\lambda$ and $\mu$ such that

$$
\left\{\begin{array}{l}
\mathcal{A} x_{*}^{p-1} y_{*}^{q}=\lambda x_{*}^{[p-1]}, \\
\mathcal{A} x_{*}^{p} y_{*}^{q-1}=\mu y_{*}^{[q-1]}, \\
\sum_{i=1}^{m}\left(x_{*}\right)_{i}^{p}=1, \\
\sum_{j=1}^{n}\left(y_{*}\right)_{j}^{q}=1,
\end{array}\right.
$$

and then we have $\lambda=\mu=\lambda_{*}=\mathcal{A} x_{*}^{p} y_{*}^{q}$. Therefore, $\lambda_{*}, x_{*}$ and $y_{*}$ satisfy the conditions of Definition 1 . This proves existence.

When $p$ and $q$ are even, $\mathcal{A}$ is positive definite if and only if the optimal objective function value of (6) is positive. Suppose that every $\mathrm{V}$-singular value of $\mathcal{A}$ is positive. By the proof for (a), $\lambda_{*}, x_{*}$ and $y_{*}$ are the optimal solution of (6), and $\lambda_{*}$ is a $\mathrm{V}$-singular value of $\mathcal{A}$. That is to say, $\lambda_{*}>0$. Hence, $\mathcal{A}$ is positive definite.

On the other hand, suppose that $\mathcal{A}$ is positive definite. Let $\lambda$ be a V-singular value of $\mathcal{A}$, $x$ and $y$ be a left and right eigenvectors of $\mathcal{A}$ associated with $\lambda$. If $f(x, y)>0$, by Theorem 2, we have $\lambda>0$.

The proof is completed.

Two sufficient conditions for the positive definiteness of partially symmetric rectangular tensors are obtained. First, we need the following lemma.

Lemma 2 Suppose that $\mathcal{A} \in \mathbb{R}^{[p ; q ; m ; n]}$ is a partially symmetric rectangular tensor, and $p$ and $q$ are even. If $\mathcal{A}$ is positive definite, then

$$
a_{i \ldots i j . . . j}>0, \quad i \in[m], j \in[n] .
$$

Proof Let $e_{i}$ be an $m$-dimensional real vector, whose $i$ th entry is 1 and others are 0 , also let $e_{j}$ be an $n$-dimensional real vector, whose $j$ th entry is 1 and others are 0 . Since the partially symmetric rectangular tensor $\mathcal{A}$ is positive definite,

$$
\mathcal{A} e_{i} \cdots e_{i} e_{j} \cdots e_{j}=a_{i \ldots i j \ldots j}>0
$$

for any $i \in[m], j \in[n]$.

Theorem 8 Let $\mathcal{A} \in \mathbb{R}^{[p ; ; ; m ; n]}$ be a partially symmetric rectangular tensor, $p$ and $q$ even, and $a_{i . . i 1 \ldots 1}=\cdots=a_{i . . . i n \ldots n}=\alpha_{i}>0, i \in[m]$. Iffor all $i \in[m]$,

$$
\alpha_{i}>R_{i}(\mathcal{A})
$$

then $\mathcal{A}$ is positive definite.

Proof Assume that $\lambda \leq 0$ is a V-singular value of $\mathcal{A}$. From Theorem 3, we have $\lambda \in \Gamma_{1}(\mathcal{A})$, hence, there is $i_{0} \in[m]$ such that

$$
\left|\lambda-\alpha_{i_{0}}\right| \leq R_{i_{0}}(\mathcal{A})
$$


From $\alpha_{i_{0}}>0$ for all $i_{0} \in[m]$, we get

$$
\left|\lambda-\alpha_{i_{0}}\right| \geq \alpha_{i_{0}}>R_{i_{0}}(\mathcal{A})
$$

This is a contradiction. Hence, $\lambda>0$. Then, the partially symmetric rectangular tensor $\mathcal{A}$ is positive definite.

Similarly to the proof of Theorem 8 , we can get the following result.

Theorem 9 Let $\mathcal{A} \in \mathbb{R}^{[p ; q ; m ; n]}$ be a partially symmetric rectangular tensor, $p$ and $q$ even, and $a_{1 \ldots 1 j . . . j}=\cdots=a_{m \ldots m j . . . j}=\beta_{j}>0, j \in[n]$. If for all $j \in[n]$,

$$
\beta_{j}>C_{j}(\mathcal{A})
$$

then $\mathcal{A}$ is positive definite.

Theorem 10 Let $\mathcal{A} \in \mathbb{R}^{[p ; q ; m ; n]}$ be a partially symmetric rectangular tensor with positive diagonal entries, $p$ and $q$ even, and

$$
\min _{i \in[m]}\left\{a_{i \ldots i 1 \ldots 1}, \ldots, a_{i \ldots . \ldots n \ldots n}\right\}=\alpha_{i}, \quad \min _{j \in[n]}\left\{a_{1 \ldots 1 \ldots 1 \ldots j}, \ldots, a_{m \ldots m j \ldots . . . j}\right\}=\beta_{j}
$$

Assume $\mathcal{B}_{1}$ and $\mathcal{B}_{2}$ are partially symmetric rectangular tensors whose $\left(i_{1} \ldots i_{p} j_{1} \ldots j_{q}\right)$ th entries are respectively defined as follows:

$$
\left(\mathcal{B}_{1}\right)_{i_{1} \ldots i_{p} j_{1} \ldots j_{q}}= \begin{cases}b_{i \ldots i i_{1} \ldots 1}=\cdots=b_{i \ldots i n \ldots n}=\alpha_{i}, & i \in[m], \\ a_{i_{1} \ldots j_{p} j_{1} \ldots j_{q}}, & \text { otherwise }\end{cases}
$$

and

$$
\left(\mathcal{B}_{2}\right)_{i_{1} \ldots i_{p} j_{1} \ldots j_{q}}= \begin{cases}b_{1 \ldots 1 j \ldots j}=\cdots=b_{m \ldots m j \ldots j}=\beta_{j}, & j \in[n], \\ a_{i_{1} \ldots j_{p} j_{1} \ldots j_{q}}, & \text { otherwise. }\end{cases}
$$

If $\mathcal{B}_{1}\left(\right.$ or $\mathcal{B}_{2}$ ) is positive definite, then $\mathcal{A}$ is positive definite.

Proof Let $x \in \mathbb{R}^{m}, y \in \mathbb{R}^{n}$ be two nonzero vectors. Since $\mathcal{B}_{1}$ is positive definite, from the definition of positive definite partially symmetric rectangular tensor, we have

$$
\mathcal{B}_{1} x^{p} y^{q}>0
$$

Then, we have

$$
\mathcal{A} x^{p} y^{q}=\mathcal{B}_{1} x^{p} y^{q}+\sum_{i \in[m], j \in[n]}\left(a_{i \ldots i j \ldots . . j}-b_{i \ldots . . i j \ldots . . j}\right) x_{i}^{p} y_{j}^{q}>0
$$

Thus $\mathcal{A}$ is positive definite. 
If $\mathcal{B}_{2}$ is positive definite, then

$$
\mathcal{B}_{2} x^{p} y^{q}>0
$$

Then, we have

$$
\mathcal{A} x^{p} y^{q}=\mathcal{B}_{2} x^{p} y^{q}+\sum_{i \in[m], j \in[n]}\left(a_{i \ldots i \ldots j . . . j}-b_{i \ldots i j \ldots . . . j}\right) x_{i}^{p} y_{j}^{q}>0
$$

Thus $\mathcal{A}$ is positive definite.

By Theorem 8, 9 and 10, we have the following sufficient conditions for the positive definiteness of partially symmetric rectangular tensors.

Theorem 11 Let $\mathcal{A} \in \mathbb{R}^{[p ; q ; m ; n]}$ be a partially symmetric rectangular tensor with positive diagonal entries, $p$ and $q$ even, and

$$
\min _{i \in[m]}\left\{a_{i \ldots i 1 \ldots 1}, \ldots, a_{i \ldots i n \ldots n}\right\}=\alpha_{i}, \quad \min _{j \in[n]}\left\{a_{1 \ldots 1 \ldots j \ldots j}, \ldots, a_{m \ldots m j \ldots . . .}\right\}=\beta_{j}
$$

If for all $i \in[m], j \in[n]$,

$$
\alpha_{i}>R_{i}(\mathcal{A})
$$

or

$$
\beta_{j}>C_{j}(\mathcal{A})
$$

then $\mathcal{A}$ is positive definite.

Based on the above theorem, we introduce the definition of strictly diagonally dominated (SDD) partially symmetric rectangular tensors.

Definition 4 Let $\mathcal{A} \in \mathbb{R}^{[p ; q ; m ; n]}$ be a partially symmetric rectangular tensor with positive diagonal entries, $p$ and $q$ even, and

$$
\min _{i \in[m]}\left\{a_{i \ldots i 1 \ldots 1}, \ldots, a_{i \ldots \ldots i n \ldots n}\right\}=\alpha_{i}, \quad \min _{j \in[n]}\left\{a_{1 \ldots 1 . \ldots j}, \ldots, a_{m \ldots m j \ldots j}\right\}=\beta_{j}
$$

Then, the partially symmetric rectangular tensor $\mathcal{A}$ is called strictly diagonally dominated (SDD), if for all $i \in[m], j \in[n]$,

$$
\alpha_{i}>R_{i}(\mathcal{A}) \text {, }
$$

or

$$
\beta_{j}>C_{j}(\mathcal{A}) .
$$


Example 4 Let us consider the rectangular tensor $\mathcal{A} \in \mathbb{R}^{[2 ; 4 ; 2 ; 2]}$ with

$$
\begin{aligned}
& a_{111111}=a_{112222}=a_{221111}=a_{222222}=1.1, \\
& a_{121111}=a_{122222}=a_{211111}=a_{212222}=-1,
\end{aligned}
$$

and other $a_{i_{1} i_{2} j_{1} \ldots j_{4}}=0$.

It can be verified that $\mathcal{A}$ satisfies all the conditions of Theorem 11, i.e., $\mathcal{A}$ is an SDD partially symmetric rectangular tensor. Hence, from Theorem $11, \mathcal{A}$ is a positive definite rectangular tensor. In fact, the $\mathrm{V}$-singular values of $\mathcal{A}$ are 2.1 and 0.1 . From the definition of positive definite partially symmetric rectangular tensors, $\mathcal{A}$ is positive definite.

Example 5 Let us consider the rectangular tensor $\mathcal{A} \in \mathbb{R}^{[2 ; 6 ; 2 ; 2]}$ with

$$
\begin{aligned}
& a_{11111111}=a_{11222222}=a_{22111111}=a_{2222222}=2, \\
& a_{12111111}=a_{12222222}=a_{21111111}=a_{21222222}=1.9,
\end{aligned}
$$

and other $a_{i_{1} i_{2} j_{1} \ldots j_{6}}=0$.

It can be verified that $\mathcal{A}$ satisfies all the conditions of Theorem 11, i.e., $\mathcal{A}$ is an SDD partially symmetric rectangular tensor. Hence, from Theorem $11, \mathcal{A}$ is a positive definite rectangular tensor. In fact, the $\mathrm{V}$-singular values of $\mathcal{A}$ are 3.9 and 0.1 . From the definition of positive definite partially symmetric rectangular tensors, $\mathcal{A}$ is positive definite.

\section{Conclusions}

In this paper, we introduce the definition of $\mathrm{V}$-singular value for rectangular tensors and obtain some $\mathrm{V}$-singular value inclusion sets for rectangular tensors with positive diagonal entries, an upper bound for the largest $\mathrm{V}$-singular value of nonnegative rectangular tensors is also given. As applications, some sufficient conditions for the positive definiteness of partially symmetric rectangular tensors are obtained. These conditions only depend on the elements of partially symmetric rectangular tensors and thus can be easily verified. At last, we introduce the definition of SDD partially symmetric rectangular tensor and show that the SDD partially symmetric rectangular tensor is positive definite.

\section{Acknowledgements}

The author would like to thank anonymous referees for encouraging and critical comments and suggestions that definitely led to improvements of the original manuscript.

\section{Funding}

This work is supported by National Natural Science Foundations of China (11661084, 71461027); Science and Technology Foundation of Guizhou province (Qian Ke He Ji Chu [2016]1161, [2017]1201); Guizhou Province Natural Science Foundation in China (Qian Jiao He KY [2016]255); Innovative talent team in Guizhou Province (Qian Ke He Pingtai Rencai [2016]5619); High-level innovative talents of Guizhou Province (Zun Ke He Ren Cai [2017]8); The doctoral scientific research foundation of Zunyi Normal College (BS [2015]09).

Availability of data and materials

The datasets generated during and/or analyzed during the current study are available from the corresponding author upon request.

Competing interests

The authors declare that they have no competing interests. 
Author details

'School of Mathematics, Zunyi Normal College, Zunyi, P.R. China. ${ }^{2}$ School of Applied Mathematics, Chengdu University of Information Technology, Chengdu, P.R. China.

\section{Publisher's Note}

Springer Nature remains neutral with regard to jurisdictional claims in published maps and institutional affiliations.

Received: 23 November 2018 Accepted: 21 March 2019 Published online: 29 March 2019

\section{References}

1. Chang, K., Qi, L., Zhou, G.: Singular values of a real rectangular tensor. J. Math. Anal. Appl. 370, 284-294 (2010)

2. Zhou, G., Caccetta, L., Qi, L.: Convergence of an algorithm for the largest singular value of a nonnegative rectangular tensor. Linear Algebra Appl. 438, 959-968 (2013)

3. Zhang, L.: Linear convergence of an algorithm for largest singular value of a nonnegative rectangular tensor. Front. Math. China 8, 141-153 (2013)

4. Ling, C., Qi, L.: / ${ }^{k, s}$-Singular values and spectral radius of rectangular tensors. Front. Math. China 8, 63-83 (2013)

5. Chirita, S., Danescu, A., Ciarletta, M.: On the strong ellipticity of the anisotropic linearly elastic materials. J. Elast. 87 $1-27(2007)$

6. Han, D., Dai, H., Qi, L.: Conditions for strong ellipticity of anisotropic elastic materials. J. Elast. 97, 1-13 (2009)

7. Qi, L.: Eigenvalues of a real supersymmetric tensor. J. Symb. Comput. 40, 1302-1324 (2005)

8. Qi, L., Luo, Z.: Tensor Analysis Spectral Theory and Special Tensors. SIAM, Philadelphia (2017)

9. Li, C., Li, Y., Kong, X.: New eigenvalue inclusion sets for tensors. Numer. Linear Algebra Appl. 21, 39-50 (2014)

10. Li, C., Li, Y.: An eigenvalue localization set for tensors with applications to determine the positive (semi-)definiteness of tensors. Linear Multilinear Algebra 64, 587-601 (2016)

11. Li, C., Zhou, J., Li, Y.: A new Brauer-type eigenvalue localization set for tensors. Linear Multilinear Algebra 64, 727-736 (2016)

12. Li, C., Jiao, A., Li, Y.: An s-type eigenvalue localization set for tensors. Linear Algebra Appl. 493,469-483 (2016)

13. Zhao, J., Li, C.: Singular value inclusion sets for rectangular tensors. Linear Multilinear Algebra 66, 1333-1350 (2018)

14. Zhao, J.: Two new singular value inclusion sets for rectangular tensors. Linear Multilinear Algebra (2018)

15. Yao, H., Zhang, C., Lei, L., Bu, C.: Singular value inclusion sets of rectangular tensors. Linear Algebra Appl. (2018)

16. Wang, Y., Qi, L., Zhang, X.: A practical method for computing the largest m-eigenvalue of a fourth-order partially symmetric tensor. Numer. Linear Algebra Appl. 16, 589-601 (2009)

17. Chang, K., Pearson, K., Zhang, T.: Perron-Frobenius theorem for nonnegative tensors. Front. Math. China 6, 507-520 (2008)

18. Yang, Y., Yang, Q.: Singular values of nonnegative rectangular tensors. Front. Math. China 6, 263-378 (2011)

\section{Submit your manuscript to a SpringerOpen ${ }^{\circ}$ journal and benefit from:}

- Convenient online submission

- Rigorous peer review

Open access: articles freely available online

- High visibility within the field

- Retaining the copyright to your article

Submit your next manuscript at $\gg$ springeropen.com 